\title{
Neoliberalismo y protección social en América Latina: salvando el capital y destruyendo el social
}

\author{
Rodrigo Cristiano Diehl ${ }^{1}$ \\ http://orcid.org/0000-0001-5675-2947
}

\author{
Jussara Maria Rosa Mendes ${ }^{2}$ \\ https://orcid.org/0000-0002-3908-4964
}

${ }^{1}$ Universidade de Santa Cruz do Sul, Departamento de Ciências Jurídicas, Programa de Pós-Graduação em Direito, Santa Cruz do Sul, RS, Brasil

${ }^{2}$ Universidade Federal do Rio Grande do Sul, Departamento de Serviço Social, Programa de Pós-Graduação em Serviço Social e Política Social, Porto Alegre, RS, Brasil

\begin{abstract}
Neoliberalismo y protección social en América Latina: salvando el capital y destruyendo el social Resumen: Conjeturar el Estado, neoliberalismo, sistemas de protección social y los movimientos políticos y económicos en América Latina en el escenario actual es un desafío provocador. Aun así, el objetivo de este estudio es comprender el Estado de orientación neoliberal en América Latina y sus impactos en los sistemas de protección social. Dado este contexto de investigación, el problema de pesquisa es: ¿cuáles son las perspectivas que deben plantearse para comprender el Estado de orientación neoliberal en América Latina con respecto a los impactos en los sistemas de protección social? Para permitir el análisis de tal escenario, el artículo se dividió en dos momentos: el primero responsable por comprender el Estado de orientación neoliberal en América Latina y, el segundo, para analizar los sistemas de protección social y las influencias del neoliberalismo en su constitución. La metodología aplicada en la investigación se divide en tres ejes: para lograr los objetivos inicialmente propuestos se utilizará investigación exploratoria-descriptiva; para organizar y recopilar los materiales para el estudio, se utilizará la investigación bibliográfica cuantitativa y cualitativa y; para el tratamiento de estos datos, el método a emplear será el materialismo-dialéctico.
\end{abstract}

Palabras clave: América Latina. Capital. Estado. Neoliberalismo. Protección social.

\section{Neoliberalismo e proteção social na América Latina: salvando o capital e destruindo o social}

Resumo: Conjecturar Estado, neoliberalismo, sistemas de proteção social e movimentos políticos e econômicos da América Latina no cenário atual, é um desafio provocador. Mesmo assim, o objetivo deste estudo é compreender o Estado de orientação neoliberal na América Latina e seus impactos nos sistemas de proteção social. Diante desse contexto de estudo, o problema de pesquisa é: quais são as perspectivas que devem ser consideradas para entender o Estado de orientação neoliberal na América Latina em relação aos impactos nos sistemas de proteção social? Para permitir a análise desse cenário, o artigo foi dividido em dois momentos: o primeiro responsável por compreender o Estado de orientação neoliberal na América Latina e o segundo por analisar os sistemas de proteção social e as influências do neoliberalismo em sua constituição. A metodologia aplicada na pesquisa está dividida em três eixos: a pesquisa exploratório-descritiva será utilizada para atingir os objetivos propostos inicialmente; para organizar e compilar os materiais para o estudo, será utilizada pesquisa bibliográfica quantitativa e qualitativa e; para o tratamento desses dados, o método a ser utilizado será o materialismo dialético.

Palavras-chave: América Latina. Capital. Estado. Neoliberalismo. Proteção social.

\section{Neoliberalism and social protection in Latin America: saving capital and destroying the social}

Abstract: Conjecturing the State, neoliberalism, social protection systems and political and economic movements in Latin America in the current scenario is a challenging challenge. Even so, the objective of this study is to understand the neo-liberal state in Latin America and its impacts on social protection systems. Given this context of study, the research problem is: what are the perspectives that must be considered to understand the neoliberal oriented State in Latin America in relation to the impacts on social protection systems? To allow the analysis of this scenario, the article was divided into two moments: the first responsible for understanding the neo-liberal state in Latin America and the second for analyzing the social protection systems and the influences of neoliberalism in its constitution. The methodology applied in the research is divided into three axes: the exploratory-descriptive research will be used to achieve the objectives initially proposed; to organize and compile the materials for the study, quantitative and qualitative bibliographic research will be used and; for the treatment of these data, the method to be used will be dialectical materialism.

Keywords: Capital. Latin America. Neoliberalism. Social protection. 
Recibido en 02.10.2019. Aprobado en 11.02.2020. Revisado en 16.03.2020.

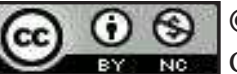

(C) El(Los) Autor(es). 2020 Acceso Abierto Esta obra está licenciada bajo los términos de la Licencia Creative Commons Atribución-NoComercial 4.0 Internacional (https://creativecommons.org/licenses/by-nc/4.0/deed.es), que permite copiar, distribuir y reproducir en cualquier medio, así como también adaptar, transformar y crear a partir de este material, desde que para fines no comerciales, y que usted fornezca el crédito debido a los autores y a la fuente, insiera un enlace para la Licencia Creative Commons e indique si fueron hechas alteraciones. 


\section{Introducción}

El capital debe entenderse como la unidad diferenciada de las relaciones sociales que se presentan en forma de explotación y dominación. Derivado de este punto, en el mundo del capital toda la relación de dominio de clase es una relación igualmente de explotación y toda la relación de explotación es, en lo que a él respecta, una relación de dominio de clases. Sin embargo, debe señalarse que existen diferentes formas de opresión y de ejercicio del poder que no constituyen en clases, por ejemplo: padre/hijo, profesor/alumno, médico/paciente, entre otras, que no afectan las formas de explotación directa sobre los trabajadores activos e indirecta sobre trabajadores inactivos.

Así, en sociedades que tienen como marca central amplias desigualdades sociales y económicas y la falta de acceso de la mayoría de la población a los sistemas de protección social a través de la garantía de los derechos sociales, como es el caso de las latinoamericanas, el estudio sobre el papel del Estado de orientación neoliberal es de suma importancia para establecerse, o al menos teóricamente, como el agente responsable por concretizar los derechos y alejar el intento de dominación del capital periférico de los principales espacios de poder y decisión.

En virtud de representar una de las principales fuentes de provisión de bienestar social en la sociedad latinoamericana, los sistemas públicos de protección social son contradictoriamente el objetivo de las políticas neoliberales ya conocidas, pero ahora travestidas con otros fenómenos (neoconservadurismo, por ejemplo), que promueven su desmonte y su descaracterización lo que implica, directamente, tanto en la concretización de los derechos de ciudadanía como en la expansión de las vulnerabilidades.

Dado este contexto, el objetivo de este estudio es comprender el Estado de orientación neoliberal en América Latina y sus impactos en los sistemas de protección social. Para lograr este objetivo, el artículo se dividió en dos momentos, que corresponden, respectivamente, a los objetivos específicos: el primero responsable por comprender el Estado de orientación neoliberal en América Latina, especialmente la necesidad de salvar el sistema capitalista, y el segundo, analizar los sistemas de protección social y las influencias directas e indirectas del neoliberalismo en su constitución.

En la construcción del trabajo, la vía metodológica utilizada en relación con los objetivos fue una investigación exploratoria-descriptiva, con respecto a los procedimientos, una investigación bibliográfica cuanticuali y, a su vez, el análisis de datos utilizó el método materialismo-dialéctico para permitir aproximación a los fenómenos naturales y sociales desde el punto dialéctico, realizando su interpretación, su forma de enfocarlos, en la perspectiva de materializar un movimiento real, sus contradicciones y fuerzas.

Por lo tanto, la pregunta es: ¿cuáles son las perspectivas que se deben plantear para comprender el Estado de orientación neoliberal en América Latina con respecto a los impactos en los sistemas de protección social?

\section{Estado de orientación neoliberal en américa latina: salvando el capital}

Al desentrañar algunos de los procesos que son fundamentales en la creación del imaginario sistema de capital, uno debe construir la premisa básica de que el modo de producción capitalista se presenta como una maraña de técnicas y relaciones sociales de producción organizada en torno a la acumulación con fines lucrativos, teniendo como su mediador el mercado y fundado en la generalización de la forma del producto que incluye, en este punto, la fuerza laboral (Jessop, 2013).

Sin embargo, la pérdida de control por parte del sistema de capital es una verdadera deficiencia, y es una consecuencia directa de la separación de funciones directamente vinculadas a la producción y el control sobre los procesos laborales. Esta deficiencia, según Bizerra y Gois (2014), provocó una ausencia de unidad, es decir, la falta de cohesión de los microcosmos socioeconómicos, al garantizar el pleno funcionamiento del sistema de capital, lo que obligó al Estado moderno a levantar sobre la base del metabolismo socioeconómico que a todo engole y, imperativamente, complementa algunas brechas (Mészáros, 2011).

Para garantizar las necesidades más básicas del sistema capitalista, el Estado moderno, entendido en su dinamismo histórico, se transforma y surge sobre todo ejerciendo su poder sobre las fuerzas centrífugas en el sistema reproductivo contradictoriamente estructurado. De esta manera, Mészáros (2003, p. 107-108) asegura que:

a estrutura legal do Estado moderno é uma exigência absoluta para o exercício da tirania nos locais de trabalho. Isso se deve à capacidade do Estado de sancionar e proteger o material alienado e os meios de produção (ou seja, a propriedade radicalmente separada dos produtores) e suas personificações, os controladores individuais (rigidamente comandados pelo capital) do processo de reprodução econômica. Sem esta estrutura jurídica, até os menores "microcosmos" do sistema do capital - antagonicamente 
estruturado - seriam rompidos internamente pelos desacordos constantes, anulando dessa maneira sua potencial eficiência econômica) [énfasis agregado].

Esta estructura legal, diseñada para el mantenimiento de los sistemas económicos, también debe estar vinculada a la destrucción de todas las barreras, ya sean locales, regionales o nacionales, que comprometan la expansión del proceso de acumulación ilimitada de capital, la base del capitalismo. Por lo tanto, debe haber una articulación muy bien estructurada entre el dinamismo histórico del capitalismo y los Estados nacionales, incluso si, "como un modo de reproducción y control sociometabólico [con su imperativo de circulación global], es inconcebible que tal sistema limítese a estos límites" (Mészáros, 2011, p. 111, traducción nuestra).

Basado en la estructuración del capitalismo global y las estructuras nacionales, y antes de entrar en los efectos causados por la aplicación de una de estas articulaciones - el neoliberalismo -, uno debe comprender su surgimiento en función de su contexto histórico. Así, como recuerda Anderson (1995), el fenómeno neoliberal es distinto del liberalismo clásico, presente en el siglo pasado, que emerge en un contexto marcado por un fuerte movimiento contrario a los privilegios de la nobleza y del Estado, predicando la igualdad legal entre los individuos. Su significado político estaba vinculado a una actitud más progresista y basado en fundamentos ideológicos de filosofía, economía y política (Carcanholo, 2004).

A su vez, el neoliberalismo nace tras el final de la Segunda Guerra Mundial en la región de Europa y América del Norte, países marcados hoy por la presencia del capitalismo central. Se puede analizar como una reacción teórica y política contra dos modelos de Estados: el Intervencionista y el Bienestar Social. Encuentra su fundamento en la teoría económica, ya que vincula todas las dimensiones de la vida y de la sociedad (social, legal, política, etc.) a criterios exclusivamente económicos, por lo que es la única opción posible. Para que su aplicación sea completa, es necesario dirigir una fuerza reaccionaria contra el Estado de Bienestar para terminar o minimizar lo más posible los logros de la clase trabajadora (Burginski, 2017).

Basado en la obra El camino de la servidumbre de Friedrich August von Hayek (1944), esta trataba-se de eliminar cualquier limitación por parte del Estado a los mecanismos de mercado al presentar una amenaza tanto para la libertad económica como para la política sujeta a intervención. Al promover un ataque en la víspera de las elecciones en Inglaterra en 1945, donde el Partido Laborista Inglés era el favorito en la carrera parlamentaria, Hayek declaró: "apesar de suas boas intenções, a socialdemocracia moderada inglesa conduz ao mesmo desastre que o nazismo alemão - uma servidão moderna” (Anderson, 1995, p. 7).

A partir de ese momento, se desarrollaron varios centros de estudio para ampliar el pensamiento de las teorías neoliberales. A seguir, lista-se las tres escuelas principales por orden de nacimiento: I) la Escuela Austriaca, dirigida por Friedrich Hayek, considerada la mecenas del pensamiento neoliberal; II) Chicago School, desarrollada por tres pensadores: Theodore William Schultz y Gari Stanley Becker (intelectuales de la teoría del capital humano) y Milton Friedman (más conocido por los medios de comunicación) y; III) Escuela de Virginia o public choice, personificada en la persona de James M. Buchanan Jr. (Moraes, 2001).

Las décadas de 1970 y 1980 se clasifican como los primeros períodos de la difusión de las ideas neoliberales del mundo a través de los procesos de globalización. Los elementos constitutivos de esta difusión se pueden agrupar en dos puntos: primero, el colapso del modelo de bienestar social y la necesidad de restaurar el poder de clase de las élites económicas que, en parte, estaban limitadas por las regulaciones estatales. Un ejemplo de este momento en América Latina fue el golpe militar en Chile (1973) que enumeró al neoliberalismo como la única salida posible a la crisis (Harvey, 2008).

El segundo factor que contribuyó decisivamente a la hegemonía neoliberal fue la desaparición de la Unión Soviética (en 8 de diciembre de 1991 con la firma del Pacto de Belaveja), que se materializó en la imaginación de un mundo unipolar (un solo polo de poder). Este hito también puede analizarse como el elemento fundamental para la derrota ideológica de la izquierda en el mundo, difundiendo la imaginación, según Pereira y Araújo (2018), de que no habría alternativas al pensamiento hegemónico: el sistema capitalista.

Con esto, el neoliberalismo marcó el comienzo de una nueva etapa dentro del sistema capitalista con una nueva (o antigua, solo recubierto) ofensiva contra los derechos sociales conquistados por los trabajadores. El Estado, dentro de la estrategia de dominación de las clases propietarias de los medios de producción, reanudó el aumento de las tasas de interés (aumentando el lucro del capital rentista) y recompuso el bloque de poder dominante. Esta reanudación no solo resultó en el desmantelamiento del Estado, al principio, debido a la necesidad de usarlo para atacar de diversas maneras, desde golpes militares hasta elecciones solo formalmente democráticas, sin la participación de la población (Castelo, 2014).

Este asalto se alió con el discurso de la institución de un Estado mínimo en todas sus esferas, sin embargo, en la práctica hubo una disminución de la participación estatal en la protección de los derechos sociales a través de políticas sociales. Por lo tanto, de acuerdo con Burginski (2017), en el neoliberalismo la 
función ideal del Estado altera la historia de un Estado mínimo y abre espacio para uno Estado fuerte en la garantía de la economía de mercado y omitido en la protección de los derechos sociales.

Esta nueva estructura de regulación social surge en base a un acuerdo entre el Estado, el capital y el trabajo, en el que la garantía de los derechos sociales estaba vinculada al aumento de la productividad y, de esta manera, los derechos sociales se crearon a partir del desarrollo positivo de la economía. Incluso con este acuerdo, la capacidad del Estado para responder a las demandas de la sociedad se ha reducido con el tiempo, debido a numerosos factores internos y externos, y como resultado, la clase dominante ha exigido repetidamente un Estado social mínimo en el que "afirmava-se que suas funções deveriam ser totalmente mínimas e que se deveria deixar o desenvolvimento entregue ao mercado e a mão invisível”. (Kliksberg, 2002, p. 3839).

El crecimiento de las escuelas de pensamiento neoliberales fue especialmente alimentado por la primera gran crisis (1973) del modelo económico capitalista en la posguerra, en el que el mundo capitalista entró en una recesión económica larga y profunda, que también causó altas tasas de inflación y bajo crecimiento de la economía. A partir de ese momento, según Anderson (1995), todo cambió, el foco de ataque de las corrientes neoliberales se dirigió al poder excesivo y dañino de los sindicatos y al movimiento obrero que fueron colocados en la pared como causas de corrosión en las bases de acumulación capitalista debido a los reclamos constantes de mejores salarios y mayor actuación del Estado en la protección social.

Por lo tanto, considerando los episodios de acción y la dirección tomada por las corrientes neoliberales, es posible concluir que el neoliberalismo se constituye como una teoría de las prácticas políticas y económicas que presupone que el bienestar de los ciudadanos es fomentado por la libertad y la capacidad empresarial individual a través de la solidez de la propiedad privada, los mercados libres y el libre comercio (léase el tránsito libre al capital). Según Harvey (2008, p. 12), "o papel do Estado é criar e preservar uma estrutura institucional apropriada a essas práticas; o Estado tem de garantir, por exemplo, a qualidade e a integridade do dinheiro".

Analizando los escenarios políticos, se puede decir que la implementación de las teorías neoliberales fue facilitada por los gobiernos de Margaret Thatcher en Reino Unido y Ronald Reagan en Estados Unidos, sin embargo, según Burginski (2017), fue en América Latina donde los primeros experimentos - comúnmente llamados de laboratorio - del neoliberalismo fueran experimentados especialmente en Chile durante el gobierno militar del general Augusto Pinochet.

Sin embargo, un punto que no se consideró en la implementación del neoliberalismo, especialmente en América Latina, son las repercusiones de las crisis cíclicas del capital que comenzaron a desatarse a fines de la década de 1970, con la profundización en la década de 1990, donde no solo llegaron a la esfera económica, pero también, en la legitimación de las nuevas iniciativas de refuncionalización del Estado para proporcionar una mayor expansión del capital (Therborn, 2012). Así, el thatcherismo en el Reino Unido,

Los procesos de producción y los resultados de las tensiones entre el trabajo y el capital se pueden visualizar en América Latina al mismo tiempo que se constituye como una región en formación histórica contradictoria. el reaganismo en los Estados Unidos y el pinoquismo en Chile, hicieron posible la visibilidad mundial de la doctrina neoliberal y el proyecto intelectual y moral de la burguesía, que se estructuraba bajo un grupo de trabajo de privatización, reducción del aparato estatal y el desmantelamiento de los sistemas de protección social logrados durante el Welfare State o inspirado en él, como es el caso en los países latinoamericanos.

La aplicación de las políticas neoliberales por parte de los Estados, al tiempo que promovió el empobrecimiento de la clase trabajadora, requería revisar las formas de intervención estatal en la cuestión social mismo que disfrazadas "por uma retórica sobre a liberdade individual, autonomia, responsabilidade pessoal” (Harvey, 2011, p. 16). En este momento, con la participación de nuevos actores, organizaciones internacionales, intereses burgueses y los Estados buscaron construir alternativas en el contexto de las políticas sociales, para que los principios básicos del mercado pudieran ser preservados. Entre las reformas institucionales, el aumento de la red de seguridad, el alivio de la pobreza y los pactos anticorrupción, el Consenso de Washington ha ganado seguidores y aplicaciones en América Latina (Simionatto \& Costa, 2014).

Las contrarreformas en el estado (ajuste estructura, privatización (inclusive de los sistemas de protección social) y la apertura externa, por ejemplo) comenzaron con la desregulación, la fragmentación-precarización y culminaron en la constitución del estado de tensión psicosocial como una herramienta poderosa que debilita las capacidades organizacionales y las voluntades de los trabajadores (Valencia, 2016). Este entorno está estructurado porque combina condiciones objetivas (crisis económicas, desempleo, disminución del ingreso promedio, 
explotación y precariedad de las relaciones laborales) y condiciones subjetivas (falta de conciencia de clase, angustia por la gran cantidad de desempleados y aumento de la pobreza).

Es importante la construcción de Wood (2014) en relación con el proceso de retirada del Estado en la conducción de políticas de bienestar social causadas por la aplicación de ideales neoliberales, para quienes, finalmente, fue creado una falsa impresión de declive del Estado. Sin embargo, lo que sucede en la práctica es todo lo contrario: el Estado está en el corazón de la globalización del capital y continúa desempeñando un papel esencial en la creación y el mantenimiento de las condiciones de acumulación de capital (Burginski, 2017), estando lejos de ser débil y las compañías multinacionales de ser todopoderosas, ya que los éxitos de las compañías dependen de los Estados.

Al utilizar Marx (2009) para llevar a cabo un análisis crítico, es posible darse cuenta de que en el capitalismo, especialmente en su orientación neoliberal, todo lo que los seres humanos consideraban inalienables se convirtió en objeto de cambio: virtud, opinión, ciencia, amor, conciencia, todo puede ser comercializado libremente. Estas nuevas configuraciones sociales y relacionales han ganado un nuevo elemento: la globalización, en la cual la mundialización (neoliberalismo + globalización) minimiza la construcción de un censo crítico y problematizador del capital financiero con características imperialistas.

En América Latina, la refundación neoliberal del orden colonial fue señalada trágicamente por la activación y el desarrollo de un nuevo ciclo de violencia imperialista. La cronología de este nuevo ciclo está enraizada en la brutal violencia perpetrada por los Estados, con golpes militares, en la década de 1970, el debilitamiento de la economía en la década de 1980 y la explotación, la deuda externa y los ajustes estructurales, como la privatización, la desregulación financiera y la flexibilización del trabajo en la década de 1990. Ahora las sociedades occidentales están en la etapa del capitalismo senil, donde todas las formas de violencia colonial conviven y coexisten en el mismo entorno sociohistórico: el terror de la represión y la criminalización de los movimientos sociales; la violencia de expropiación que expulsa a las poblaciones de sus territorios y; la disminución de investimentos en áreas prioritarias (Aráoz, 2013).

Esta nueva estructura, que se denomina de hegemonía neoliberal, en la que el Estado, en términos de protección y concretización de los derechos sociales, ahora solo tiene funciones residuales - como políticas compensatorias -, y salud, educación y seguridad social por el mercado. Junto con este escenario, existe la institución de un otro paradigma: la reanudación del papel del Estado como promotor de políticas sociales con el objetivo de promover la justicia social, combatir la exclusión y las desigualdades. Las nuevas políticas sociales tienen como ejes de transformación la selectividad y la ampliación de los criterios de focalización, la introducción de la participación del sector empresarial, el fortalecimiento de las asociaciones con el tercer sector y la ampliación e institucionalización de la participación social (Melo \& Paese, 2013).

Por lo tanto, teniendo como trapo de fundo la técnica presentada, en América Latina existe una fuerte tendencia a que la ciudadanía sea concebida como una contraparte del Estado en la existencia de un proceso en el cual la formulación genérica y abstracta es cruzada por un lógica del ejercicio del poder político a través del cual se reifican las estructuras de dominación particularistas/personalistas (Fleury, 1994). Sin embargo, su (de)construcción ocurre en la tensionalidad, es decir, en los límites funcionales entre la garantía de la protección social a los ciudadanos y su violación a través de constantes contrarreformas y entregas al capital.

Analizando la lógica del capitalismo contemporáneo y sus interfaces con el neoliberalismo, la tendencia no es la eliminación completa de las políticas sociales; por el contrario, es la creación de una tensión creciente de la universalidad y redistribución de los servicios públicos de protección social, especialmente, aquellos derivados de dos enfoques: I) flexibilización y precariedad de las relaciones laborales con la reducción de los encargos sociales (impuestos) para expandir las superganancias y; II) supercapitalización, es decir, la ocurrencia de privatizaciones declaradas en sectores sociales, como salud, educación y seguridad social (Behring, 2008).

En un entorno de contrarreforma, en la concepción de Behring (2008), la defensa de la universalidad de las políticas sociales debe incluso permitir el cuestionamiento de una estrategia anticapitalista, debido a la incapacidad de las reformas estructurales frente a las fuerzas del capital. Según Marx (2006), al capital no se preocupa en garantizar la salud o la vida del trabajador, a menos que los movimientos de la sociedad lo obliguen a respetarlo.

De los argumentos presentados, el que tiene más probabilidades de destruir los sistemas públicos de protección social es su vinculación al trabajo, o sea, la garantía del bienestar social sin el apego al trabajo debe minimizarse en el capitalismo. Bajo las bases del neoliberalismo, según Sampaio y Oliveira (2016), la atención del Estado a los necesitados (especialmente a los económicamente pobres) debe observar el postulado de la vinculación: protección y trabajo, para que la práctica de protección mientras un derecho de todos no sirva de desánimo o disminución de la voluntad individual al trabajo.

De esta manera, todos los que se han negado o no pueden participar (incluidos los que se han convertido en incapaces al trabajar como resultado de su propio trabajo) como mano de obra en el mercado serán de alguna manera excluidos de la construcción social. Esta exclusión también incluye a los desempleados, ya que 
el vínculo entre protección y trabajo no existirá. Por lo tanto, la pobreza y el desempleo serán considerados como procesos similares en su forma de rechazar a los hombres de la sociedad moderna más envidiable: la esfera de los bienes y los privilegios económicos (Xiberras, 1996).

Estos fenómenos deben ser considerados en América Latina dentro de una realidad social de conceptos dinámicos y mutantes, donde las transformaciones sociales que ocurrieron en la última década permitieron un cambio de perspectiva con consecuencias para los estudios sociales en los países centrales y periféricos del capitalismo mundial. Sorj (2001) enseña que la principal fuente de este cambio de perspectiva es la propia crisis de la modernidad en los países centrales: estructurales y conquistas sociales que parecían inamovibles. Las sociedades centrales (clasificadas como avanzadas) muestran graves signos de crisis, exclusión social y de violencias, en otras palabras, no hay más un punto de llegada, por lo tanto, ni conceptos sólidos con los que comparar la etapa actual de los países periféricos del capital.

Según estimaciones de la Comisión Económica para América Latina y el Caribe (CEPAL) (2019) y la Organización Internacional del Trabajo (OIT), la tasa de desempleo en América Latina es aproximadamente de $10 \%$ (diez por ciento) entre la población económicamente activa. Lo que, analizando desde una perspectiva neoliberal, al menos una décima parte de la población latinoamericana (sin considerar a otros grupos) no estaría protegida por los sistemas públicos de protección social si estuvieran directamente vinculados al trabajo, es decir, aquellos sin-sin: sin trabajo y sin protección social.

Por lo tanto, se muestra que el fenómeno sociolaboral actual también es desigual, ya que tiene varias funciones según el país analizado, esta diferencia puede verificarse a partir de algunos elementos, tales como: el grado de desarrollo económico de un país (en este punto, la cuestión de la ubicación del capital, periférico o central, implica enfoques variables) y la conciencia de la clase trabajadora y la lucha por la manutención y la expansión de los derechos fundamentales (individuales, colectivos y fraternos).

En la necesidad de vincular el trabajo y protección social, la orientación neoliberal construye la siguiente máxima: propiciai direitos aos pobres e tereis mais pobres, tentando constituir en la sociedad que el mantenimiento y/o la expansión de los sistemas de protección social refuerza un comportamiento de dependencia de los pobres con el sistema. Este intento enfrentamiento, según Sampaio y Oliveira (2016), surge de la preocupación de los conservadores en incentivar aquellos trabajadores que receben bajos salarios (o aquellos fuera del mercado laboral) a reclamar ser beneficiarios (no como sujetos con derechos) del aparato de protección social.

Además de presentarse como un pensamiento extremadamente limitado con respecto a los sistemas de protección social y las desigualdades sociales, económicas y políticas, remete la pobreza a la esfera moral. Construir al pobre como ese individuo, en el análisis ya privado de derechos, que no acertó en la vida y, por lo tanto, su pobreza económica (sin analizar los otros elementos directamente vinculados) es el resultado de su incapacidad personal de progresar o es resultado de su pereza.

Como una forma de combatir esta visión problemática de la pobreza, algunas organizaciones internacionales e incluso Estados reconocen la necesidad de abordar las manifestaciones de la cuestión social no con políticas asistencialistas, que no alteran el status quo del orden capital, pero sí, en promover debates críticos en la constitución de una emancipación. Sin embargo, Simionatto y Costa (2014) señalan que la construcción de un proyecto civilizador que atienda a todos los seres humanos solo será posible, dado el escenario actual, si la presión proviene de las clases oprimidas, es decir, de bajo de la sociedad. Marx (1975) ya estaba preocupado con el encantamiento de la clase trabajadora, ya que la constante manutención y reproducción de la clase trabajadora sigue siendo una condición para la reproducción del capital.

Llevando en consideración esta estructura estatal puesta (quizás, impuesta) de Estado (democracia), sociedad (bloque histórico) y mercado (neoliberalismo), el análisis del propio Estado latinoamericano es extremadamente importante como herramienta para comprender la estructura subyacente de cualquier política pública, en particular, los sistemas de protección social. Además de ser un proceso histórico construido por la clase trabajadora, de aceptación y de legitimación, la protección social se desarrolla a través de la garantía de los derechos de ciudadanía a favor o en contra del propio Estado, pero que, en la actualidad, es en el proceso de caracterización y desmontaje. Este será el tema del próximo capítulo.

\section{Desprotección social en América Latina hoy: destruyendo el social}

El sistema capitalista tiene la facilidad de promover conjuntamente dos fenómenos contradictorios: el avance en el campo de la producción y la considerable expansión de la pobreza, como resultado de la mala distribución de la producción socialmente producida, bajo los objetivos de la explotación laboral. Así se presenta la ley general de acumulación capitalista, donde las expresiones de la cuestión social representan el resultado del intento de confrontar las dos clases fundamentales del capital. En esta dinámica prevista 
por las relaciones entre capital y trabajo, se producen avances (reformas) o retrocesos (contrarreformas) en los sistemas de protección social.

Los procesos de producción y los resultados de las tensiones entre el trabajo y el capital se pueden visualizar en América Latina al mismo tiempo que se constituye como una región en formación histórica contradictoria. Cada uno de los veinte países ${ }^{1}$ tiene sus especificidades que no deben sublimarse y analizarse de muchas maneras como si fueran un solo bloque global. Sin embargo, cuando se realiza una inserción específica en la división internacional del trabajo, basada en una construcción histórica dentro del capitalismo mundial y, con base en Carcanholo (2014), es posible dar a la economía latinoamericana un carácter, con diferentes grados, de dependencia y ubicación periférica en la acumulación de capital. Por lo tanto, la dependencia y la periferia en el sistema económico mundial caracterizan la unidad de América Latina, sin excluir las especificidades de cada miembro.

El mantenimiento de estas dos situaciones contradictorias de formación de países explotados y explotadores es posible hoy en día mediante la creación de vínculos financieros en que los primeros (países periféricos) dependen de préstamos, incentivos fiscales, empleos, tecnología y productos básicos impuestos por los últimos (países centrales). Del mismo modo que, según Pereira (2017), los países explotados deben alentar a la fuerza laboral con baja paga, que mantiene a un gran número de personas en la pobreza, para minimizar los derechos laborales y las regulaciones, promover la privatización de los servicios públicos y hacer pactos con el gran capital central.

En el avance de las reflexiones, es imposible no mencionar la ola histórica dejada por los proyectos populares en América Latina que los movimientos de derecha actuales, junto con los medios de comunicación, el poder judicial y el parlamento, tienen la intención de eliminar de la escena política a través de ideologías hegemónicas de mantenimiento de la superestructura y del aumento de la tensión social. Incluso porque es en el campo de la moral y la cultura donde la derecha gana espacio, tanto entre la clase media como entre las clases bajas (Rojas \& Gomes, 2017).

En el ambiente latinoamericano, también se están produciendo otros cambios, donde el neoliberalismo asume la centralidad de la regulación gubernamental todos los días, promoviendo una estabilización de fuerzas que cruzan el terreno de la dominación y la dirección de un proyecto colectivo de nación. Para Rojas y Gomes (2017), América Latina está experimentando un momento turbulento en sus democracias, especialmente frente a un número considerable de medidas de austeridad ante el ajuste económico, lo que causará severas limitaciones en la actuación de los sistemas de protección social.

Los procesos de avance del conservadurismo (o neoconservadurismo) y de la propia derecha en el continente latinoamericano no pueden ser vistos y analizados como casos aislados o no conectados, sino como una unidad dialécticamente engendrada. Bering (2008) afirma que el conservadurismo político se ha mantenido constante, pero últimamente ha adquirido nuevas formas de establecer fuentes de legitimidad (neoconservadurismo) como: clientelismo moderno, neocorporatismo y movilizaciones de voluntariado y solidaridad.

Por lo tanto, analizar los fenómenos que afectan al Estado en América Latina y, en consecuencia, el desmantelamiento de los sistemas de protección social no es una tarea sencilla, especialmente cuando se contempla una reorganización de las crisis y de la clase burguesa, junto con la expansión del imperialismo y del neocolonialismo, atestiguado en la adaptación a la geopolítica mundial (Rojas \& Gomes, 2017).

Lo que está sucediendo actualmente en los sistemas de protección social no debe verse como algo nuevo, sino más bien como una mejora, con nuevos actores y formas sociales de los procesos de contrarreforma vistos desde 1980 en adelante con la expansión del capitalismo mundial con el apoyo de las agencias (Banco Mundial, Fondo Monetario Internacional y Banco Interamericano de Desarrollo, por ejemplo). Las implementaciones de contrarreforma en los Estados latinoamericanos (contrarreforma administrativa) y en la protección social (desmantelamiento de los sistemas públicos) tienen su punto de partida en el Consenso de Washington y la falacia de la necesidad de estabilización económica e inflacionaria. Estas contrarreformas, al principio, actuaron en la privatización de los servicios públicos y de las empresas públicas, es decir, redujeron el papel del Estado en la sociedad.

Junto con las políticas neoliberales, se produjo la expansión del neoconservadurismo, constituyendo, según Pereira (2017), lo que convencionalmente se llama de la Nueva Derecha. Esta Nueva Derecha une elementos del neoliberalismo como la realización del culto al libre mercado, la defensa incondicional de la privatización con apertura al capital extranjero, la reducción de la acción estatal en la esfera social y la regulación de la economía con elementos neoconservadores (patriotismo, jerarquía, orden y moral) formando nuevas tendencias culturales, sociales y políticas de carácter conservador y excluyente.

Este acercamiento del conservadurismo con las políticas neoliberales también influye en las instituciones privadas tradicionales, como la familia (patriarcal) y la iglesia, que nuevamente influyen significativamente en las esferas públicas colectivas. Sin mencionar que el resurgimiento de la ideología neoconservadora y su 
intersección con el neoliberalismo permite el surgimiento de la extrema opresión de las minorías sociales que promueven discursos misóginos, racistas, homofóbicos y xenófobos (Pereira, 2017).

Junto con estos discursos, el nuevo paradigma periférico de la pobreza como elemento central de la política social y los diseños de programas se expande en todo el mundo capitalista periférico y central. Estas nuevas políticas están diseñadas con la ayuda de organizaciones multilaterales que buscan abordar solo las expresiones y manifestaciones inmediatas del problema social (como la pobreza absoluta), perdiendo cualquier vínculo directo con problemas estructurales (como el desempleo, el racismo y el machismo). Este nuevo pensamiento y actuación, que la academia desempeña un papel importante en el mantenimiento, refuerza las acciones enfocadas e individualizadas, sin actuar sobre temas centrales que generalmente son las causas de tal realidad (Pastorini \& Martinez, 2014).

Otro punto importante en la construcción de estos nuevos modelos de acción es vincular la expansión de la pobreza con la necesidad de reducir la naturaleza protectora del Estado (a través de los sistemas públicos de protección social), lo que incluye aumentar la dimensión represiva y coercitiva: reemplazar un Estado social por un Estado punitivo. Estos cambios, tanto en la sociedad como en el Estado, solo son posibles gracias al apoyo político y económico de varios sectores de la sociedad frente a la extensión de nuevas narrativas, actores y situaciones que promueven la inseguridad y elaboran una cultura del miedo como figura omnipresente. Donde el miedo, la inseguridad y la violencia se han convertido en factores importantes de cohesión social (Morás, 2010).

El capitalismo, especialmente cuando se guía por el neoliberalismo y el neoconservadurismo, encontrase en la raíz de la existencia de la pobreza, de la miseria, de la injusticia social y de la degradación humana en sus más variadas facetas de crueldad (Alayón, 2016). Así, los sistemas de protección social se enfrentan a un tsunami de darwinismo social, que tiende a predominar en la lógica perversa del funcionamiento mundial.

En un intento de evitar, no hacer frente, esta situación desarrolla políticas sociales como políticas estatales de intervención en las condiciones de reproducción de la vida en diversas clases y grupos sociales. Así, se presenta como un campo de relaciones y prácticas, organizado por el Estado, en cuyo interior surge la disputa por la constitución de condiciones básicas de vida como un asunto público (aquí emprendido en el sentido colectivo/interés general) o como un asunto privado (Danani, 2010).

La respuesta de las políticas públicas y sociales a la coyuntura de protección social se resume actualmente en una promesa civilizadora perversa que, basada en una construcción ideológica fetichizada que establece que, a través del acceso al empleo, se enfrentarán manifestaciones de (des)protección, que se basa en la idea de que la falta de calificación profesional es un factor decisivo para la empleabilidad (Gershenson \& Scherer, 2016). Así, el proceso educativo sería el único agente en la constitución social y en el apaciguamiento de la lucha de clases, ocultando las relaciones de explotación.

Vale la pena reflexionar sobre las políticas sociales y su campo de acción más allá del horizonte de establecerse como una mera estrategia de acomodación de conflictos o caridad social. Para esto, según Paiva y Ouriques (2006), es necesario referirse a ellos dentro del proceso de disputa política para obtener un superávit económico real por parte de las clases que históricamente han sido expropiadas, y no simplemente en políticas que reducen las graves manifestaciones de pobreza (pobreza extrema, por ejemplo) a través del acceso a servicios sociales básicos.

El Estado insertado en el capitalismo monopolista de orientación neoliberal actúa como un instrumento de organización económica, pero siempre tiene como objetivo gestionar los ciclos de la crisis y proporcionar mecanismos para su renovación a expensas de la clase trabajadora. Dentro de este contexto, se promueven políticas sociales en la idea de que el Estado gestione las expresiones y manifestaciones de la cuestión social, garantizando condiciones básicas de supervivencia de la población en nombre del desarrollo monopolístico del capital (Raichelis, 2013).

Sin embargo, bajo el viejo discurso, el espacio de las políticas públicas también se está desmantelando en todo el mundo, especialmente en América Latina. En Europa, existen fuertes movimientos neoliberales y neoconservadores para desmantelar lo que ha quedado del Estado de bienestar social, por ejemplo: en la seguridad social (aumento de la edad, aumento del período de cotización), en las relaciones laborales (disminución de los derechos laborales, finalización estabilidad y endurecimiento de los requisitos de acceso al seguro de desempleo), en la asistencia social (demanda de compensación y condicionalidad para recibir prestaciones sociales), en la salud y en la educación con la introducción de la necesidad de pago de algunos servicios, desmantelando la universalidad (Boschetti, 2009).

No muy diferente del territorio latinoamericano, donde las propuestas para la construcción de nuevas direcciones se basan en la fórmula del neoliberalismo, bajo los siguientes principios: I) el desarrollo de un Estado que rompa con el poder de los sindicatos; II) un Estado con bajo desempeño en el área social y regulación económica; III) la búsqueda de la estabilidad monetaria sobre todos los demás aspectos de la vida en sociedad; IV) reestructuración del gasto social y, en consecuencia, expansión de la tasa natural de desempleo; V) reformas 
fiscales, al reducir la recaudación de impuestos de mayores ingresos y; VI) la disminución de la operatividad de los derechos sociales con el desmantelamiento de los sistemas de protección social (Behring, 2009).

\section{Consideraciones finales}

El estudio del Estado y de los sistemas públicos de protección social en América Latina se basa en la necesidad de problematizar teorías eminentemente latinoamericanas que entiendan y lleven en cuenta las especificidades y la diversidad de las naciones, así como el grave cuadro de desigualdades sociales y económicas. Bajo este nuevo contexto de propuesta, se puede decir que las sociedades latinoamericanas deben luchar contra los discursos y las acciones, especialmente las neoliberales, que tienen como objetivo reducir o incluso exterminar los sistemas de protección social.

En este contexto, es esencial percibir la explotación y el dominio como una forma de conocer la realidad, ya que el capital no puede revelarse en el mundo de los fenómenos con estas acciones. El capital tiene entre sus promesas promover un mundo con hombres libres e iguales que, incluso violando, deben reconstituirlo. Existe, por lo tanto, la creación de un mundo imaginario (ficción) que cubre y distorsiona la esencia de su ser (mundo real), donde el capital necesita presentarse de manera distorsionada, para no revelar, a primera vista, lo que es de hecho. Este fenómeno se llama fetichización del capital, que termina creando un mundo encantado: el capital como una fábula.

Bajo este escenario, el presente estudio tuvo como objetivo responder a este problema: ¿cuáles son las perspectivas que se deben plantear para comprender el Estado de orientación neoliberal en América Latina con respecto a los impactos en los sistemas de protección social? Donde, debido a este movimiento de contrarreformas en el Estado y en los sistemas de protección social que hasta entonces estaban guiados por los principios de igualdad, equidad y justicia distributiva, es un ambiente de reveses y retirada de los derechos sociales. Contradictoriamente en su lugar, las ideologías, los valores y las prácticas que se oponen a los derechos sociales ganan terreno, imponiendo límites en la realización de políticas públicas de beneficio para todos, sobre la base de una nueva administración de escasos recursos públicos.

Al mismo tiempo que la dependencia forma una mutualidad, constituye procesos contradictorios. Por un lado, hay naciones explotadas y empobrecidas con altas tasas de desigualdades sociales, económicas, políticas y de acceso a los derechos, que se componen de una fuerza poco calificada y mal remunerada y trabajan con instalaciones de subempleo, lo que ha causado, especialmente en los últimos años, la disminución del poder colectivo para exigir al Estado mejoras sociales. Por otro lado, están las naciones explotadoras y colonizadoras que se han enriquecido con el despojo de la riqueza mineral y la explotación del trabajo de esas personas y Estados que están al margen del capitalismo.

Como perspectivas para la comprensión de los Estados latinoamericanos y de los sistemas de protección social, existe, en particular, un intento de mercantilizar su propia vida en la sociedad, usurpando los derechos de ciudadanía y, por lo tanto, disminuyendo el papel y la participación del Estado en la protección social. En este punto, se afirma que la realidad, los hechos y los eventos necesitan ser desenmascarados, de modo que haya un largo camino de construcción entre la apariencia y la esencia, entre la parte y la totalidad, entre lo singular y lo universal.

\section{Referencias}

Alayón, N. (2016). Desafíos para el trabajo social en América Latina en los momentos decisivos de capital y el avance del conservadorismo. Revista Textos \& Contextos, 15, 10-18.

Anderson, P. (1995). Balanço do neoliberalismo. In E. Sader, \& P. Gentili (Orgs.), Pós-neoliberalismo: as políticas sociais e o Estado democrático (pp. 9-23). Rio de Janeiro, Brasil: Paz e Terra.

Aráoz, H. (2013). Orden neocolonial, extractivismo y ecología política de las emociones. Revista Brasileira de Sociologia da Emoção, $12,11-34$.

Behring, E. R. (2009). Acumulação capitalista, fundo público e Política Social. In I. Boschetti et al. (Org.), Política social no capitalismo: tendências contemporâneas (2a ed., pp. 13-34). São Paulo, Brasil: Cortez.

Behring, E. R. (2008). Brasil em contrarreforma: desestruturação do Estado e perda de direitos (2a ed.). São Paulo, Brasil: Cortez. Bizerra, F. A., \& Gois, J. C. S. (2014). Estado e capital: uma ineliminável relação de complementariedade à base material. Revista Katálysis, 17, 77-86.

Boschetti, I. (2009). Seguridade social na América Latina. In I. Boschetti et al. (Org.), Política social no capitalismo: tendências contemporâneas (2a ed., pp. 174-195). São Paulo, Brasil: Cortez. 
Burginski, V. M. (2017). A contrarreforma do Estado na ideologia neoestruturalista da Cepal. Revista Em Pauta, 15(40), $280-295$. Carcanholo, M. D. (2004). Neoliberalismo e desenvolvimento em uma economia periférica. In C. L. Xavier (Org.), Desenvolvimento desigual (pp. 283-320). Uberlândia, Brasil: Editora da Universidade Federal de Uberlândia.

Carcanholo, M. D. (2014). Desafios e perspectivas para a América Latina do século XXI. Revista Argumentum, 6(2), 6-24.

Castelo, R. (2014). Estado, transformismo e supremacia rentista no capitalismo dependente brasileiro contemporâneo. Revista Ser Social, 16, 296-302.

Comisión Económica para América Latina y el Caribe (2019). Panorama social de América Latina 2019. Recuperado de https:// repositorio.cepal.org/bitstream/handle/11362/44969/5/S1901133_es.pdf

Danani, C. (2010). El espacio publico en su laberinto: las políticas sociales y las confontaciones del universalismo. In N. Gluz, \& J. A. Salgado (Comp.), Particularismo y universalismo en las políticas sociales: el caso de la educación (pp. 59-76). Buenos Aires, Argentina: Universidad Nacional de General Sarmiento.

Fleury, S. (1994). Estado sem cidadãos: seguridade social na América Latina. Rio de Janeiro, Brasil: Fiocruz.

Gershenson, B., \& Scherer, G. A. (2016). Uma promessa civilizatória perversa: as políticas públicas e juventudes na era neodesenvolvimentista. Revista Textos \& Contextos, 15, 160-170.

Harvey, D. (2011). O enigma do capital: e as crises do capitalismo. São Paulo, Brasil: Boitempo.

Harvey, D. (2008). O neoliberalismo: história e implicações. São Paulo, Brasil: Loyola.

Jessop, B. (2013). Política social, Estado e "sociedade”. Revista Ser Social, 15, 261-384.

Kliksberg, B. (2002). Repensando o estado para o desenvolvimento social: superando dogmas e convencionalismos (2a ed.). São Paulo, Brasil: Cortez.

Marx, K. (2006). El capital (7a ed.). México: Fondo de Cultura Económica.

Marx, K. (1975). Prefácio à contribuição à crítica da economia política. In Marx, K., \& Engels, F. Textos 3 (pp. 82-83). São Paulo, Brasil: Edições Sociais.

Marx, K., \& Engels, F. (2009). A ideologia alemã. São Paulo, Brasil: Expressão Popular.

Melo, L. V., \& Paese, C. R. (2013). As políticas sociais e o combate à exclusão: repensando o papel do Estado na contemporaneidade. Revista Argumentum, 5, 102-119.

Mészáros, I. (2003). O século XXI: socialismo ou barbárie? São Paulo, Brasil: Boitempo.

Mészáros, I. (2011). Para além do capital: rumo a uma teoria da transição. São Paulo, Brasil: Boitempo.

Moraes, R. C. (2001). Neoliberalismo: de onde vem, para onde vai? São Paulo, Brasil: Senac.

Morás, L. E. (2010). Los dilemas y desafíos de las políticas progresistas de seguridad. Revista Encuentros Uruguayos, (3), 6-21.

Paiva, B. A., \& Ouriques, N. (2006). Uma perspectiva latino-americana para as políticas sociais: quão distante está o horizonte? Revista Katálysis, 9, 166-175.

Pastorini, A., \& Martínez, I. (2014). Tendências das mudanças da proteção social no Brasil e no Uruguai: a centralidade das redes mínimas na América Latina. Revista Katálysis, 17, 59-67.

Pereira, C. P. (2017). A proteção social em tempos sem brios. Revista Argumetum, 9, $24-29$.

Pereira, V. S., \& Araújo, M. A. (2018). Rupturas, neogolpismo e América Latina: uma análise sobre Honduras, Paraguai e Brasil. Revista Katálysis, 21, 125-136.

Raichelis, R. (2013). Proteção social e trabalho do assistente social. Tendências e disputas na conjuntura de crise mundial. Revista Serviço Social e Sociedade, São Paulo, (116), 609-635.

Rojas, G. A., \& Gomes, C. M. C. (2017). Crise orgânica, governos e seus impactos na América Latina. Revista Em Pauta, 39(15), $17-32$. Sampaio, S. S., \& Oliveira, R. (2016). Estado-Providência: chega de odes e críticas neoliberais. Revista Serviço Social e Sociedade, (126), 302-317.

Simionatto, I., \& Costa, C. R. (2014). Estado e políticas sociais: a hegemonia burguesa e as formas contemporâneas de dominação. Revista Katálysis, 17, 68-76.

Sorj, B. (2001). A nova sociedade brasileira. Rio de Janeiro, Brasil: Jorge Zahar.

Therborn, G. (2012). Do marxismo ao pós-marxismo? São Paulo, Brasil: Boitempo.

Valencia, A. S. (2016). Dependência, precariedade laboral e fratura social na América Latina com as reformas neoliberais. Revista Argumentum, 8, 140-152.

Wood, E. M. (2014). O império do capital. São Paulo, Brasil: Boitempo.

Xiberras, M. (1996). As teorias da exclusão: para a construção do imaginário do desvio. Lisboa, Portugal: Instituto Piaget.

\section{Nota}

1 Argentina, Bolivia, Brasil, Chile, Colombia, Costa Rica, Cuba, Ecuador, El Salvador, Guatemala, Haití, Honduras, México, Nicaragua, Panamá, Paraguay, Perú, República Dominicana, Uruguay y Venezuela. 


\section{Rodrigo Cristiano Diehl}

rodrigocristianodiehl@live.com

Máster en Derecho, Universidade de Santa Cruz do Sul (UNISC)

Máster en Política Social y Trabajo Social por la Universidade Federal de Rio Grande do Sul (UFRGS)

\section{UNISC}

Av. Independência, n. 2293 - Bairro Universitário

Santa Cruz do Sul - RS - Brasil

CEP: $96815-900$

\section{Jussara Maria Rosa Mendes}

jussaramaria.mendes@gmail.com

Doctora en Trabajo Social por la Pontifícia Universidade Católica de São Paulo (PUC-SP)

Profesora Acreditada al Programa de Posgrado en Política Social y Trabajo Social, Universidade Federal do Rio Grande do Sul (UFGRS).

\section{UFRGS}

Av. Paulo Gama, n. 110 - Bairro Farroupilha

Porto Alegre - RS - Brasil

CEP: $90040-060$

\section{Agradecimientos}

Este trabajo es resultado de las discusiones proporcionadas por el Núcleo de Estudios en Salud y Trabajo vinculado al Programa de Posgrado en Política Social y Trabajo Social de la Universidade Federal de Rio Grande do Sul.

\section{Agencia financiadora}

No se aplica.

\section{Contribuciones de los autores}

Rodrigo Cristiano Diehl (autor y revisor); Jussara Maria Rosa Mendes (autora y orientadora).

\author{
Aprobación por Comisión de Ética y consentimiento para \\ participación \\ No se aplica.

\section{Consentimiento para publicación} \\ Consentimento dos autores. \\ Conflicto de intereses \\ No hay conflicto de intereses.
}

